\title{
Bond or band?
}

\section{Is there a difference in clinical failure rates between bonded and banded first molar attachments during fixed appliance therapy?}

\author{
Banks P, Macfarlane TV. \\ Bonded versus banded first molar attachments: a randomized \\ controlled clinical trial. J Orthod 2007; 34:128-136
}

Design This was a prospective randomised controlled clinical trial (RCT). Intervention The experimental group received single first molar tubes bonded with a no-mix chemically cured composite (Rely-a-Bond; Reliance, Itasca, Illinois, USA) after a 30-second etch. The control group participants were treated with bands cemented with glass ionomer cement (Intact; Ortho-Care, Bradford, West Yorkshire, UK).

Outcome measure The primary outcome was first-time attachment failure (tooth level) and the secondary outcome was number of first-time failures per patient (patient level).

Results There were $18.8 \%$ first-time failures in the band group compared with $33.7 \%$ in the bonded group. Bonds had a relative risk of failure of 2.4 (95\% confidence interval, 1.4-4.1) compared with bands. There was no difference in failure rates between maxillary and mandibular teeth, or between left and right sides. Experimental group patients also had more bracket failures (P 0.009), when analysed at patient level. Conclusions First molar tubes bonded with Rely-A-Bond composite showed a significantly higher first-time failure rate than bands cemented with Intact glass-ionomer cement.

\section{Commentary}

Orthodontists have been examining the need for bonding molar attachments. The advantages are plenty: it has been theorised in the literature that the very act of placing separators tends to sensitise the periodontal ligament, setting in motion the cellular processes responsible for orthodontic tooth movement and possibly compromising anchorage, even before loading in extraction treatment. The initial pain on placement of separators requires medication and, in cases of anticipated bacteraemia, antibiotic cover may be necessary. Economy of space in nonextraction treatment is also crucial.

A study such as this is likely to receive much critical attention from the orthodontic fraternity, who would rather see their apprehensions about molar bonding set to rest rather than affirmed. The prospective RCT, however, has all the hallmarks of careful, well-structured design and methods. The aim is stated as an investigation of failure rates between bonded and banded molar attachments. The inclusion and exclusion criteria, clinical protocols, randomisation and outcome assessment all pass muster easily. The experimental and control groups are well balanced. The statistical analysis is stringent and allows comparison with previous published data, and standard errors have been adjusted to prevent a bias in reporting results. The authors have been fairly critical and conclusive about the limitations of the study in terms of the sample size.

The overall failure rates are quoted as $18.8 \%$ for the bands and $33.7 \%$ for the bonds, with no difference between maxillary and mandibular molars and right and left sides. This is in slight contradiction of published literature, since previous studies report higher failures on the righthand side. Where the figures seem likely to starkly affect the decision-making of the clinician, however, is the $34 \%$ failure rate of molar bonding: should an orthodontist even think about bonding molars? In a cost-benefit analysis, this could outweigh all the possible advantages. Herein lies the conundrum of drawing conclusions from a good RCT like this one.

There are plainly plenty of reasons for failure of bonds, from operator skill, the size of the bondable base to the type of adhesive. The no-mix adhesives, such as the one used in the study, work on the principal of inhomogeneous polymerisation wherein a catalyst gradient is set up from the primed enamel surface towards the bracket base by diffusion. Such adhesives, however, have the disadvantage of decreased resin strength because of a disturbed crosslinkage network. This can be compounded by the fact that manipulation of a tube into position on a molar is difficult and a small shift in the tube before polymerisation is complete may decrease the bond strength. The outcome and clinical implications would therefore diverge from the intention of the study to discover the failure rates between bonded and banded molar attachments.

The authors have correctly concluded that the clinical implications of the failure of bonding molar attachments in this study lie with this specific Rely-a-Bond composite. Orthodontists may take heart from the fact that good work has been done with moisture-insensitive and newer light-cured adhesives, greatly improving bonding of attachments to molars. The take-home message is implicit in the caution of the authors statement that failures were multifactorial, ranging from the attachment, patients' social circumstances to operator skills.

\section{Anmol Kalha}

Department of Orthodontics and Dentofacial Orthopaedics, College of Dental Sciences, Davangere, Karnataka, India

1. Eliades T. Orthodontic materials research and applications. Part 1. Current status and projected future developments in bonding and adhesives. Am J Orthod Dentofacial Orthop 2006; 130:445-451.

2. Millett DT, Hallgren A, Fornell AC, Robertson M. Molar bonded tubes: a retrospective evaluation of clinical performance. Am J Orthod Dentofacial Orthop 1999; 165:667-674.

Evidence-Based Dentistry (2007) 8, 105. doi:10.1038/sj.ebd.6400524 